\title{
Minimally invasive esophagectomy and radical lymph node dissection without recurrent laryngeal nerve paralysis
}

\author{
Koji Otsuka ${ }^{1}$ (D) Masahiko Murakami ${ }^{1}$ - Satoru Goto ${ }^{1} \cdot$ Tomotake Ariyoshi $^{1} \cdot$ Takeshi Yamashita $^{1} \cdot$ Akira Saito $^{1}$. \\ Masahiro Kohmoto ${ }^{1} \cdot$ Rei Kato $^{1} \cdot$ Alan Kawarai Lefor ${ }^{2} \cdot$ Takeshi Aoki $^{1}$
}

Received: 15 April 2019 / Accepted: 4 January 2020 / Published online: 3 February 2020

(c) The Author(s) 2020

\begin{abstract}
Background We introduce a novel operative technique to dissect lymph nodes adjacent to the recurrent laryngeal nerve, referred to as the "native tissue preservation" technique. Using this technique, there was no damage to the recurrent laryngeal nerve, which is maintained in its anatomical position.

Methods From September 2016 to December 2018, minimally invasive esophagectomy was performed in the left lateral decubitus position in 87 patients with esophageal cancer. The native tissue preservation technique for lymphadenectomy around the recurrent laryngeal nerve was used, and all patients were evaluated for recurrent laryngeal nerve paralysis.

Results Minimally invasive esophagectomy was completed in all patients without conversion to thoracotomy. Although an extended lymphadenectomy was performed in all patients, there were no grade II or higher complications (Clavien-Dindo classification) and no incidence of recurrent laryngeal nerve paralysis.

Conclusion The native tissue preservation technique may reduce the incidence of recurrent laryngeal nerve paralysis after minimally invasive esophagectomy with radical lymph node dissection.
\end{abstract}

Keywords Minimally invasive esophagectomy $\cdot$ Radical lymphadenectomy $\cdot$ Recurrent laryngeal nerve paralysis $\cdot$ Prevent complications $\cdot$ Native tissue preservation $\cdot$ Micro-anatomical layer

Recurrent laryngeal nerve paralysis (RLNP) causes incomplete closure of the vocal cord folds and limits the ability to successfully cough, as the patient cannot develop sufficient pressure. The risk of pulmonary complications is greatly increased in patients with RLNP because of an increased likelihood of aspiration and the development of aspiration pneumonia [1]. RLNP is considered a major postoperative

This study was submitted for EAES 2019, was nominated for the Best Video Presentation Award.

Electronic supplementary material The online version of this article (https://doi.org/10.1007/s00464-020-07372-3) contains supplementary material, which is available to authorized users.

Koji Otsuka

kojiotsuka@med.showa-u.ac.jp

1 Department of Surgery, Division of Gastroenterological and General Surgery, School of Medicine, Showa University, 1-5-8 Hatanodai, Shinagawa-ku, Tokyo 142-8666, Japan

2 Department of Surgery, Jichi Medical University, Tochigi, Japan complication of esophageal surgery. Although it depends on the extent of lymph node dissection, the reported incidence of RLNP after esophagectomy varies from 8.3 to $40.9 \%$ [2-4].

We previously reported our procedure for thoracoscopic esophagectomy in the left lateral decubitus position and suggested the benefits of minimally invasive esophagectomy and use of the technique to limit morbidity [5]. Intraoperative nerve injury is caused by thermal damage, stretching, cutting, compression, and vascular compromise to the nerve. These mechanisms more frequently affect the left recurrent laryngeal nerve compared to the right, because of its longer course and vulnerability within the mediastinum. Minimally invasive esophagectomy is particularly beneficial to reduce the incidence of postoperative respiratory complications, but may be associated with a higher incidence of left recurrent nerve palsy according to one large Japanese study [6]. In this study, we present our lymph node dissection technique, with specific attention to the micro-anatomical layer to avoid recurrent laryngeal nerve injury. 


\section{Materials and methods}

\section{Study design and patient characteristics}

We previously reported our procedure and outcomes of thoracoscopic esophagectomy in the left lateral decubitus position [5] and have performed over 1100 minimally invasive esophagectomy procedures over the last 21 years. From May 2016 to December 2018, thoracoscopic resection for patients with esophageal cancer in the left lateral decubitus position was attempted in 87 patients by the same surgical team at Showa University Hospital. Inclusion criteria were patients with carcinoma of the thoracic esophagus, without serious cardiac or respiratory disease that would preclude safe conduct of surgery under general anesthesia, without metastases to other organs such as the lung or liver, and tumor stage lower than Stage T4b. Clinicopathological factors were classified according to the UICC-TNM (7th edition) criteria [7]. Surgical outcomes data were collected, and use of these data was approved by the Institutional Review Board of Showa University, Tokyo, Japan.

\section{Surgical procedure}

\section{Anesthesia, positioning, and port placement}

Surgery was performed after induction of general anesthesia. In general, two-lung ventilation was used during the thoracoscopic and abdominal portions of the procedure. One-lung pulmonary ventilation with an 8-Fr spiral tube was occasionally used, and the right bronchus was occluded when two-lung ventilation was difficult. The thoracic portion of the operation was performed in the left lateral decubitus position with $15^{\circ}$ head elevation. The surgeon stood on the dorsal side of the patient, and a high-definition video monitor was placed at the patient's head. The first observation port $(12 \mathrm{~mm})$ was placed at the 5th intercostal space in the anterior axillary line. Carbon dioxide pneumothorax was achieved at a pressure of $8 \mathrm{~mm} \mathrm{Hg}$, and the right lung was decompressed. This was followed by placement of 5-mm ports for the operator in the fifth and eighth intercostal spaces at the posterior axillary line. A 5-mm port for the thoracoscope was inserted into the eighth intercostal region at the mid-axillary line, and $12-\mathrm{mm}$ ports for the assistant were inserted in a slightly ventral position in the third intercostal space at the anterior axillary line (Fig. 1).

\section{Dissection technique}

\section{Right recurrent laryngeal nerve lymph node}

The lymph nodes around the right recurrent laryngeal nerve were resected. Since three to four branches run from the right

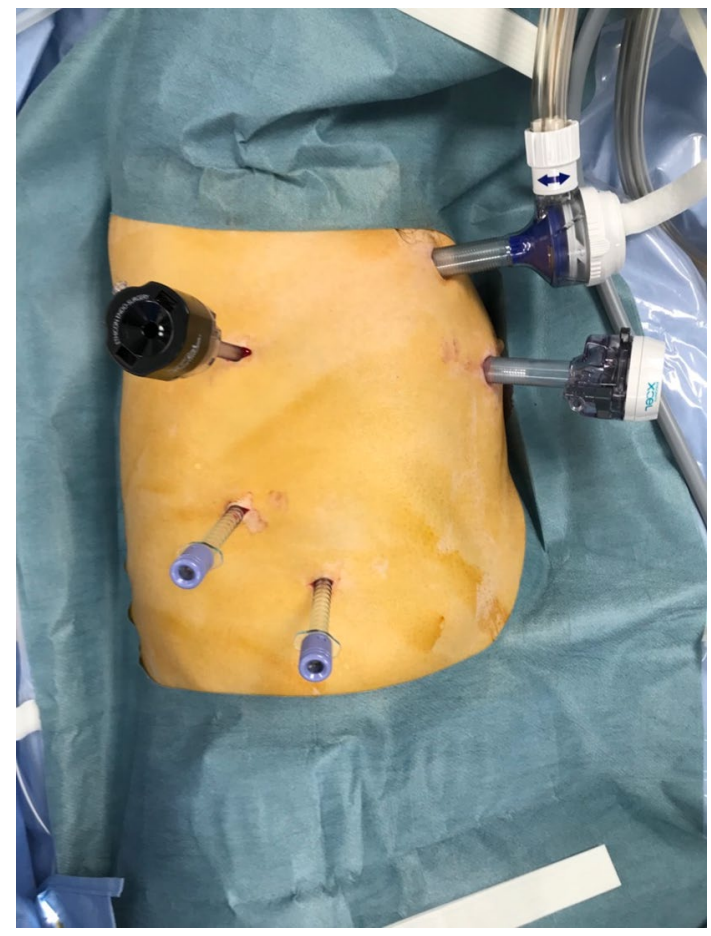

Fig. 1 Port placement: three 5-mm ports and two 12-mm ports are used

recurrent laryngeal nerve toward the esophagus, these were divided with an ultrasonic device (HARMONIC ACE+7, Johnson and Johnson, New Brunswick, NJ, USA). In this dissection, we preserve the thin layer of connective tissue around the right recurrent laryngeal nerve, paying careful attention to the risk of thermal damage and avoiding traction on the nerve when dissecting the right recurrent laryngeal nerve lymph node (Fig. 2). We also preserve the thin native tissue overlaying the right recurrent laryngeal nerve.

\section{Left recurrent laryngeal nerve lymph node on the dorsal side of the trachea}

We dissected the area between the trachea and esophagus using an ultrasonic instrument. First, we dissected the ventral side with adipose tissue including the left recurrent laryngeal nerve and lymph nodes until we confirmed the overlying shiny thin layer of connective tissue. This shiny thin layer is on the cardiac branch of the sympathetic nerve and is preserved to avoid cardiac arrhythmias (Fig. 3). After dissection inferior to the aortic arch to the cervicothoracic junction, we dissected around the proximal thoracic esophagus, and the esophagus was transected using an automatic suture device (Echelon Gold $60 \mathrm{~mm}$; Johnson and Johnson). By rotating the trachea toward the ventral side, we obtained a good view of the adipose tissue, which contains the left 


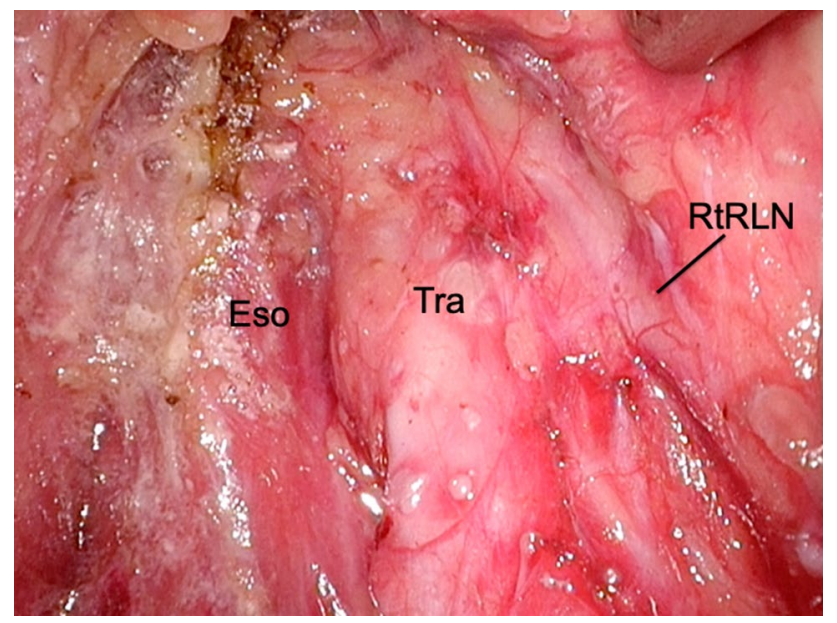

Fig. 2 View after dissection around the right recurrent laryngeal nerve lymph node: we preserve the thin layer of connective tissue around the right recurrent laryngeal nerve. Eso esophagus, RtRLN right recurrent laryngeal nerve, Tra trachea

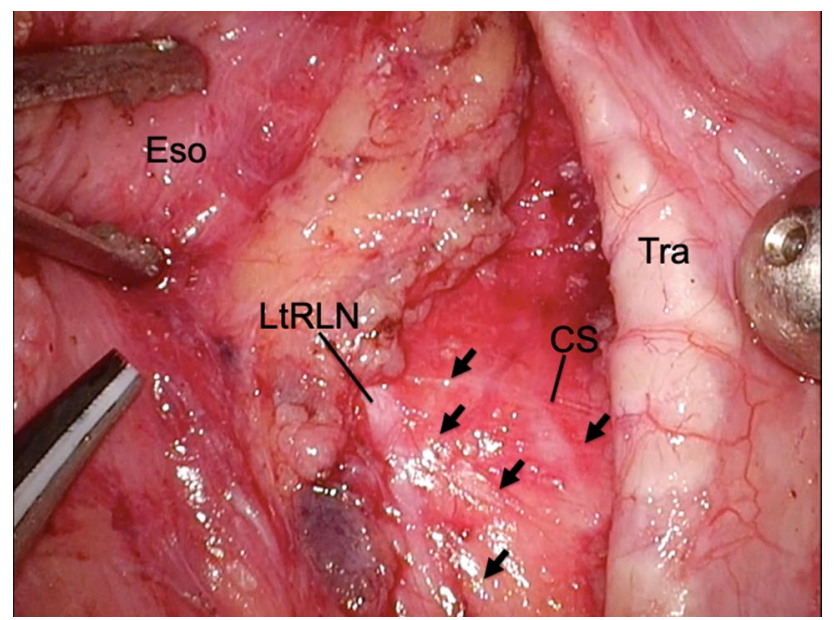

Fig. 3 Dissection view around the left recurrent laryngeal nerve and cardiac branch of the sympathetic nerve: we dissect the ventral side with fatty tissue including the left recurrent laryngeal nerve and lymph nodes until we can confirm the overlying shiny thin layer of connective tissue. This shiny thin layer is on the cardiac branch of the sympathetic nerve and is preserved to avoid cardiac arrhythmias. Black arrows show the shiny thin layer. $C S$ cardiac branch of the sympathetic nerve, Eso esophagus, LtRLN left recurrent laryngeal nerve, Tra trachea

recurrent laryngeal nerve and lymph nodes, without the esophagus.

\section{Native tissue preservation technique of the recurrent laryngeal nerve}

The aim of this technique is to prevent RLNP based on the premise that by not actually touching the nerve, traction and injury to the recurrent laryngeal nerve are limited while maintaining the normal anatomical position of the left recurrent laryngeal nerve. We rotate the left recurrent laryngeal lymph nodes and adipose tissue counterclockwise from the ventral side to the dorsal side about the axis of the left recurrent laryngeal nerve (Fig. 4). By preserving the thin native tissue layer around the left recurrent laryngeal nerve, traction and bending are avoided (Fig. 5A, B). We carefully avoid grasping the adipose tissue, avoiding traction on the nerve, and not touching the nerve when dissecting around the left recurrent laryngeal nerve lymph nodes. We mainly use an ultrasonic dissector, avoiding thermal damage while dissecting around the left recurrent laryngeal nerve. We limit the time of application of the ultrasonic instrument and use a short pitch dissection technique, limited to 1-2 s. Division of a few transverse vessels around the left recurrent laryngeal nerve was confirmed after ultrasonic dissection. Recently, we have used the HARMONIC HD 1000i Shears (Johnson and Johnson) during minimally invasive esophagectomy, which allows dissection without using scissors around the recurrent laryngeal nerve and its branches. After the adipose tissue and lymph nodes are rotated dorsally to the left recurrent laryngeal nerve, we confirm the location of the left recurrent laryngeal nerve under the thin layer of connective tissue. The overlying adipose tissue and lymph nodes on the superior aspect are dissected, and the area marked with a clip to facilitate confirmation of the location during the cervical procedure (Fig. 6). These lymph nodes were dissected from caudal to cranial because there are small branches of the left recurrent laryngeal nerve passing from caudal to cranial, and these branches should be divided in the same direction to avoid injury to the

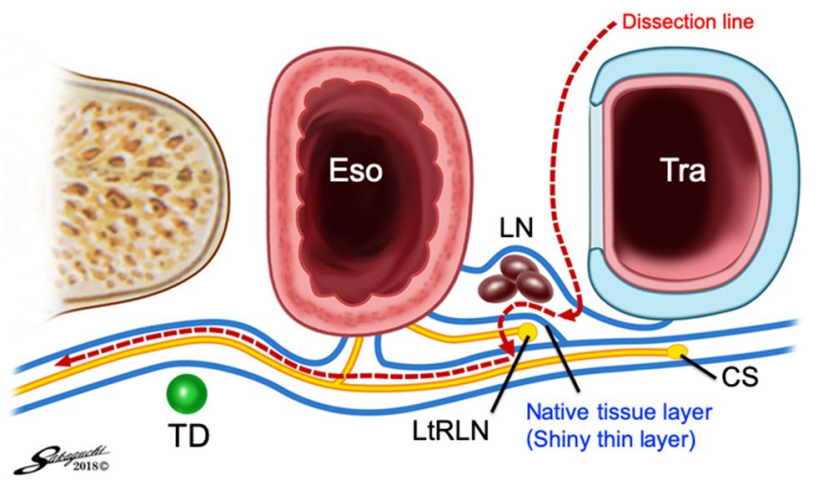

Fig. 4 Coronal view of the dissection field: first, we dissect the dorsal side of the trachea. Then, the left recurrent laryngeal lymph nodes and adipose tissue are rotated counterclockwise from the ventral side to the dorsal side about the axis of the left recurrent laryngeal nerve. The blue line shows the native tissue layer (shiny thin layer) located above the left recurrent laryngeal and the cardiac branch of the sympathetic nerve. Eso esophagus, $C S$ cardiac branch of sympathetic nerve, $L N$ lymph node, $L t R L N$ left recurrent laryngeal nerve, $T D$ thoracic duct, Tra trachea (Color figure online) 

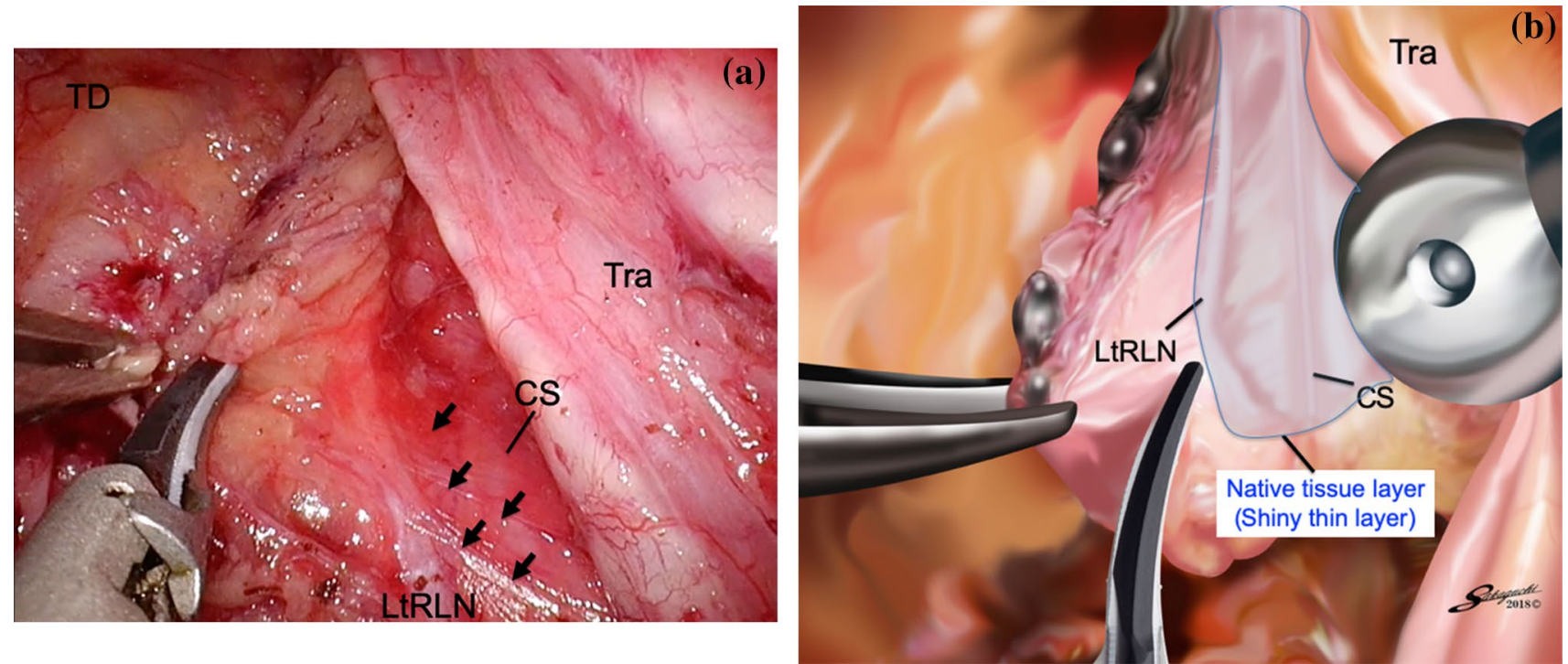

Fig. 5 A Dissection view around the left recurrent laryngeal nerve and cardiac branch of the sympathetic nerve without showing the esophagus: the overlying thin layer of native tissue is identified, and the lymph nodes and fatty tissue are rotated counterclockwise from the ventral side to the dorsal side about the axis of the left recurrent laryngeal nerve. Black arrows show the shiny thin layer. B The left

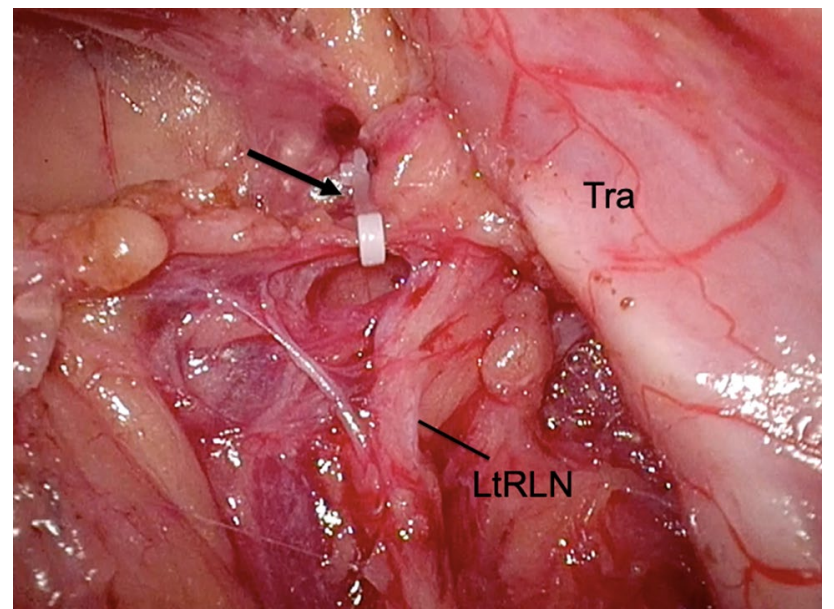

Fig. 6 Dissection view of the superior side around the left recurrent laryngeal nerve. A clip (arrow) is placed to facilitate confirmation of the location during the cervical procedure. $L t R L N$ left recurrent laryngeal nerve, Tra trachea

main nerve. We can then safely dissect the left recurrent laryngeal nerve lymph nodes that are already rotated toward the dorsal side of the left recurrent laryngeal nerve with an adequate margin from the nerve (Fig. 7). The left recurrent laryngeal nerve lymph nodes are removed while maintaining the normal anatomical position of the left recurrent laryngeal nerve without touching and stretching the nerve (video). The intrathoracic superior landmark recurrent laryngeal nerve and lymph node are observed clearly after esophageal dissection. The native tissue layer (shiny thin layer) is observed above the left recurrent laryngeal nerve and cardiac branch of the sympathetic nerve (blue area); we should preserve this layer. $L t R L N$ left recurrent laryngeal nerve, $C S$ cardiac branch of the sympathetic nerve, $T D$ thoracic duct, Tra trachea (Color figure online)

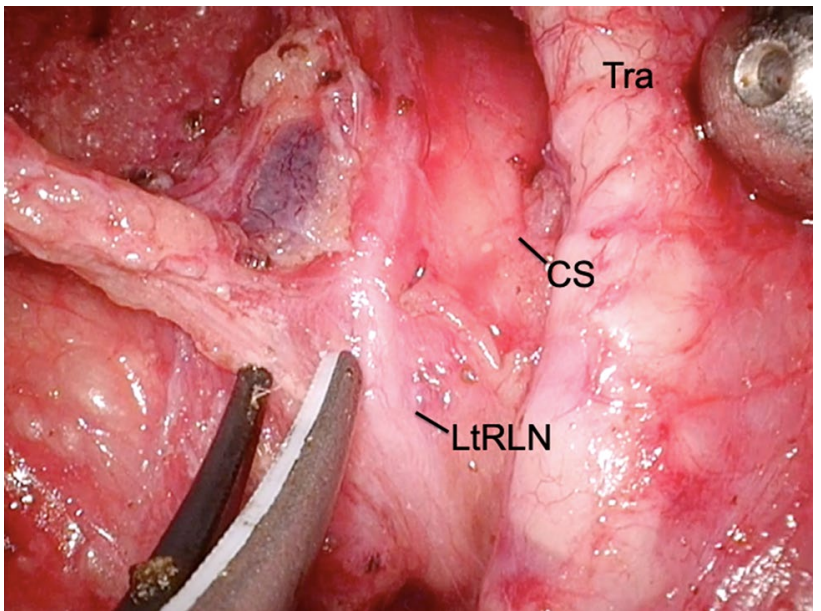

Fig. 7 Native tissue preservation technique: the left recurrent laryngeal nerve lymph nodes are safely dissected after rotation along the dorsal side of the left recurrent laryngeal nerve with an adequate margin between the area of dissection and the nerve without touching and stretching the nerve. $C S$ cardiac branch of the sympathetic nerve, $L t R L N$ left recurrent laryngeal nerve, Tra trachea

clip placed on the left recurrent laryngeal nerve lymph node can help confirm the location of the lymph node and provide information on how much of the lymph node can be dissected during the cervical procedure (Fig. 8). 


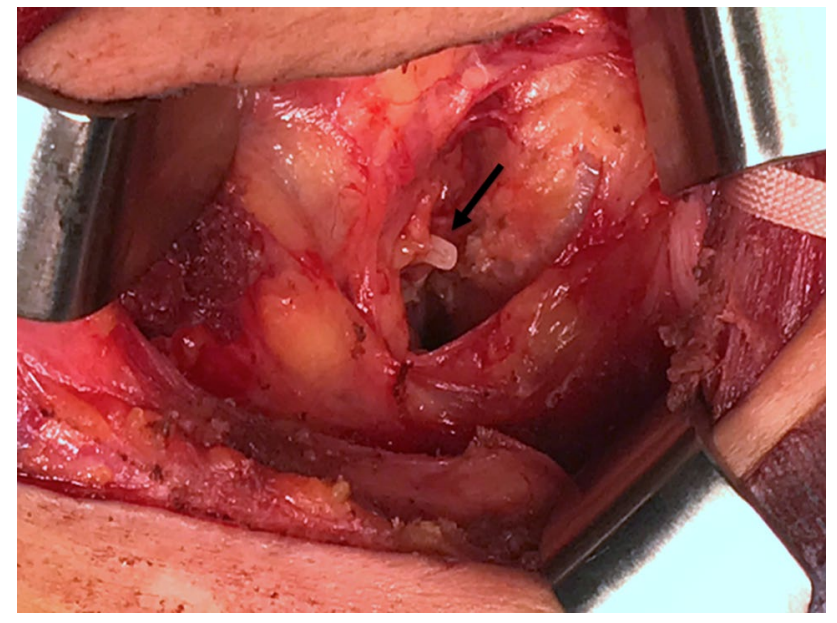

Fig. 8 Cervical procedure view: intrathoracic superior landmark clip of the left recurrent laryngeal nerve lymph node facilitate confirmation of its location during cervical procedure (black arrow)

\section{Evaluation for RLNP}

The presence of RLNP was assessed by the anesthesiologist at extubation and assessed by an endoscopist and an otolaryngologist using a flexible laryngoscope before starting oral intake (postoperative day 5) regardless of the presence or absence of hoarseness or paralysis. We also evaluate all patients for hoarseness until discharge. The grade of RLNP was classified according to the Clavien-Dindo classification [8] and supplementary criteria advocated by the Japan Clinical Oncology Group [9]. The definition of RLNP was any classification higher than grade II. If a patient was found to have RLNP, an otolaryngologist performed a follow-up examination. After discharge, patients were continuously followed up regarding hoarseness of their voice once every month in the first 6 months and every 3 months thereafter at the outpatient clinic. These data were collected by review of medical records.

\section{Results}

Demographic characteristics for all patients are shown in Table 1 . The mean age was 64.9 years and the study group included $75 \%$ men. Tumors were located in the cervical (2.3\%), upper thoracic (14\%), middle thoracic (46\%), lower thoracic (29\%), and abdominal (9.2\%) esophagus. Squamous cell carcinoma was diagnosed in $87 \%$, adenocarcinoma in 9.2\%, and carcinosarcoma in $2.3 \%$ of patients on the basis of histologic findings. Preoperative therapy was administered to $93 \%$ of patients. Neoadjuvant chemotherapy was administered to $89 \%$ of patients. Four patients $(4.6 \%)$ with suspected cT4 lesions were administered neoadjuvant chemo-radiation
Table 1 Patient characteristics $(N=87)$

\begin{tabular}{ll}
\hline Age, mean (years) & 64.9 \\
Male, $n(\%)$ & $65(75)$ \\
Tumor location & \\
Cervical esophagus, $n(\%)$ & $2(2.3)$ \\
Upper esophagus, $n(\%)$ & $12(14)$ \\
Middle esophagus, $n(\%)$ & $40(46)$ \\
Lower esophagus, $n(\%)$ & $25(29)$ \\
Abdominal esophagus, $n(\%)$ & $8(9.2)$ \\
Histologic findings & \\
Squamous cell carcinoma, $n(\%)$ & $76(87)$ \\
Adenocarcinoma, $n(\%)$ & $8(9.2)$ \\
Carcinosarcoma, $n(\%)$ & $2(2.3)$ \\
Neuroendocrine carcinoma & $1(1.1)$ \\
pTNM stage & \\
Stage IA, $n(\%)$ & $28(3.2)$ \\
Stage IB, $n(\%)$ & $3(3.4)$ \\
Stage IIA, $n(\%)$ & $11(13)$ \\
Stage IIB, $n(\%)$ & $17(19)$ \\
Stage IIIA, $n(\%)$ & $15(17)$ \\
Stage IIIB, $n(\%)$ & $6(6.9)$ \\
Stage IIIC, $n(\%)$ & $7(8.0)$ \\
Stage IV, $n(\%)$ & $0(0)$ \\
Neoadjuvant therapy & \\
None, $n(\%)$ & $38(44)$ \\
Chemotherapy, $n(\%)$ & $49(56)$ \\
Chemo-radiation, $n(\%)$ & $6(6.9)$ \\
Pleural adhesions & $77(89)$ \\
Yes, $n(\%)$ & $4(4.6)$ \\
No, $n(\%)$ & $28(32)$ \\
Number of lymphadenectomy fields & $59(68)$ \\
Two, $n(\%)$ & \\
Three, $n(\%)$ & \\
Anastomosis site & \\
Cervical, $n(\%)$ & \\
Intrathoracic, $n(\%)$ & \\
Conversion to thoracotomy, $n(\%)$ & \\
\hline
\end{tabular}

therapy. Lung adhesions were noted during surgery in 32\% of patients. Thoracoscopic esophagectomy was completed in all patients without conversion to open thoracotomy.

Surgical outcomes and complications are shown in Table 2. There were no blood transfusions due to thoracoscopic blood loss. The mean number of thoracic lymph nodes retrieved was 26.5 , and the mean number for all lymph nodes was 58.6. The overall complication rate was $20 \%$ including anastomotic leak in $3.4 \%$ and postoperative bleeding in $2.3 \%$ of cases. There were no instances of grade II or higher postoperative RLNP (Clavien-Dindo classification). However, temporary hoarseness (Clavien-Dindo grade I) was observed in two patients $(2.3 \%)$ who recovered 
Table 2 Surgical outcomes and postoperative complications $(\mathrm{n}=87)$

\begin{tabular}{ll}
\hline Thoracic operative time (min), mean & 183.7 \\
Thoracic blood loss (ml), mean & 57.6 \\
Blood transfusion (ml), mean & 0 \\
Extubation (days after surgery), mean & 0 \\
Postoperative length of stay (days), mean & 16.3 \\
Number of retrieved thoracic lymph nodes, mean & 26.5 \\
Number of retrieved total lymph nodes, mean & 58.6 \\
Overall complication rate, $n(\%)$ & $18(20)$ \\
Surgical complications & \\
Chylothorax, $n(\%)$ & $0(0)$ \\
Anastomotic leakage, $n(\%)$ & $3(3.4)$ \\
Postoperative bleeding, $n(\%)$ & $2(2.3)$ \\
Recurrent laryngeal nerve paralysis, $n(\%)$ & $0(0)$ \\
Non-surgical complications & \\
Arrhythmia, $n(\%)$ & $4(4.6)$ \\
Pneumonia, $n(\%)$ & $3(3.4)$ \\
Pulmonary thrombosis, $n(\%)$ & $1(1.1)$ \\
Atelectasis, $n(\%)$ & $1(1.1)$ \\
Reoperation within 30 days, $n(\%)$ & $2(2.3)$ \\
Mortality within 30 days, $n(\%)$ & $0(0)$ \\
\hline
\end{tabular}

without specific therapy within 2 months. Arrhythmias were observed in $4.6 \%$ of patients, pulmonary thrombosis in $1.1 \%$, atelectasis in $1.1 \%$, and pneumonia in $3.4 \%$. Reoperation within 30 days was necessary in $2.3 \%$ of patients due to postoperative bleeding. There was no mortality within 30 days.

\section{Discussion}

By avoiding direct nerve injury, unreasonable traction, thermal damage, and other factors associated with RLNP, the incidence of RLNP should be minimized. Herein, we have reviewed the dissection techniques used to limit the occurrence of RLNP, with specific focus on the anatomical layer around the recurrent laryngeal nerve. This new technique for limiting the incidence of RLNP is based on the simple concept of preserving the thin layer of tissue on the left recurrent laryngeal nerve. We refer to this as the native tissue preservation technique. Although we dissected the recurrent laryngeal nerve lymph nodes bilaterally, we did not note any incidence of RLNP (Clavien-Dindo classification > grade II) in this series of patients. This novel technique enabled an extended radical lymphadenectomy with retrieval of a mean of 26.5 lymph nodes from the mediastinum and 58.6 total lymph nodes.

Thoracic esophageal cancer accounts for approximately $90 \%$ of esophageal cancers in Japan, with a relatively high risk of metastases to upper mediastinal lymph nodes along the recurrent laryngeal nerves bilaterally [10]. Moreover, we clinically suspected metastasis in the recurrent laryngeal nerve lymph nodes in 51 cases $(58.6 \%)$. Right recurrent laryngeal nerve lymph node metastasis was noted in 47 cases (54\%) and left recurrent laryngeal nerve lymph node metastasis was noted in 19 cases (21.8\%); bilateral recurrent laryngeal nerve lymph node metastasis was observed in 15 cases $(17.2 \%)$ in our study. We chose neoadjuvant chemotherapy for $89 \%$ cases in this study, and a total of 24 cases $(27.6 \%)$ of lymph node metastasis around the recurrent laryngeal nerve was diagnosed. Based on the pathological findings, lymph node metastasis on the right side was detected in 18 cases $(20.7 \%$ ), while lymph node metastasis on the left side was detected in 12 cases (13.8\%); bilateral metastasis was detected in 6 cases $(6.9 \%)$. Given the nature of the disease, extended radical lymphadenectomy including the upper mediastinal lymph nodes along both recurrent laryngeal nerves is considered a standard surgical approach in Japan [11-13]. Although it depends on the extent of lymph node dissection performed, the incidence of RLNP after esophagectomy with radical lymph node dissection is reported to range from 8.3 to $40.9 \%$ [2-4]. This rate of RLNP after a three-field lymph node dissection is unacceptably high following esophagectomy. Radical lymphadenectomy including the upper mediastinal nodes after neoadjuvant chemotherapy for patients with advanced esophageal cancer improved the overall 5-year survival rate from 50 to $70 \%$ in our institution [5].

The resection of esophageal cancer has recently been performed thoracoscopically for many patients [6]. We use thoracoscopic magnification during lymph node dissection and divide the branches of the recurrent laryngeal nerve carefully to avoid thermal damage, stretching, cutting, compression, and vascular compromise. Placing a tape around the recurrent laryngeal nerve during lymphadenectomy is usually performed during radical esophagectomy performed through a thoracotomy $[11,13]$. However, minimally invasive esophagectomy has a higher rate of recurrent nerve palsy compared with open surgery (10.3\% vs. $8.1 \%)$, suggesting the need to more carefully avoid RLNP when performing resection of esophageal cancer [6]. Minimally invasive esophagectomy has good outcomes usually with little bleeding, but we noticed thermal damage and stretching of the recurrent laryngeal nerve during the lymphadenectomy. We believe these factors contribute to the development of RLNP and care must be taken to avoid these actions intraoperatively.

Most dissections were performed with an ultrasonic surgical device when performing the upper mediastinal lymphadenectomy along the recurrent laryngeal nerves during minimally invasive esophagectomy. Special care is taken to avoid thermal damage and cavitation. There is evidence that the use of electrosurgical devices may lead to inadvertent damage to nearby structures such as the bowel, nerves, or 
blood vessels through the lateral spread of thermal energy [14-17]. Studies also show a correlation of the degree of thermal injury with lateral thermal spread $[18,19]$. A study of the Harmonic Scalpel device demonstrated that it may be used without a substantial rise in the temperature of adjacent tissues. An increase in tissue temperature above $42{ }^{\circ} \mathrm{C}$ results in both damage to cell membranes and protein denaturation [20]. Sutton et al. suggested that the use of electrosurgical instruments is associated with a significant rise in temperature at the tip of the instrument, proportional to the power and length of time of application [21]. We are vigilant regarding the duration of application and use the short pitch dissection technique within 1-2 s when dissecting connective tissue and small vessels. These precautions may limit thermal damage to the recurrent laryngeal nerve.

To investigate the influence of distance from the instrument on damage to the recurrent laryngeal nerve, Megan et al. compared thermal spread and recurrent laryngeal nerve function with the THUNDERBEAT Open Fine Jaw device, the bipolar Ligasure Small Jaw, and the ultrasonic Harmonic Focus for open thyroidectomy [22]. This study concluded that RLN injury did not occur if the devices were used approximately $2 \mathrm{~mm}$ away from the nerve. We did not maintain this $2 \mathrm{~mm}$ margin from the nerve because of existing adipose and lymph node tissue that must be radically resected inside that margin. Although we cannot conclude a specific safe distance from the nerve based on this study, we believe that attention to meticulous dissection during lymphadenectomy around the nerve with magnification can avoid RLNP due to thermal damage.

Stretching of the recurrent laryngeal nerve during lymphadenectomy must be avoided. We believe that excessive stretching is the most common mechanism of recurrent laryngeal nerve injury. The left recurrent laryngeal nerve is more frequently involved, probably because the longer course of the nerve creates additional vulnerability, especially within the mediastinum. However, this native tissue preservation technique was used around not only the left recurrent laryngeal nerve area but also the right recurrent laryngeal nerve lymph node. Thyroid surgeons suggest that excessive traction is the most common mechanism of recurrent laryngeal nerve injury and suggest that intraoperative nerve monitoring may be a useful adjunct to decrease the rate of injury [23-25]. We used intraoperative nerve monitoring in the past, but it was difficult to prevent injury because the monitoring was not continuous. We could only determine the incidence of recurrent laryngeal nerve palsy, but continuous intraoperative nerve monitoring was useful to decrease the injury in thyroid surgery [24]. In a recent animal study, Deguchi et al. studied approaches to decrease the incidence of RLN damage during esophageal surgery [26] and suggested that continuous intraoperative nerve monitoring may be useful to decrease the incidence of nerve injury in esophageal surgery. The underlying concept of this native tissue preservation technique is to maintain the recurrent laryngeal nerve in its normal anatomical position without stretching, thermal damage, or touching the nerve, and this approach limits the incidence of RLNP, as there was no incidence of RLNP in 87 consecutive patients in this study. The native tissue preservation technique also limits the incidence of vascular compromise to the recurrent laryngeal nerve. Blood flow affects nerve function and regeneration [27, 28]. We believe that the thin layer of connective tissue around the recurrent laryngeal nerve also preserves the small blood vessels that supply the recurrent laryngeal nerve.

Although it has been suggested that neoadjuvant chemo-radiation therapy increases postoperative complications and mortality compared to surgery alone [29], we performed neoadjuvant chemo-radiation (40 Gy) followed by minimally invasive esophagectomy using the native tissue preservation technique after 4 weeks in four cases. We did not note a significant difference in the thoracic operation time (175.5 min), thoracic blood loss $(68 \mathrm{ml})$, and postoperative length of stay (16.5 days) compared to the other techniques. There were no complications in our patients. Therefore, our procedure can be performed safely for patients who receive neoadjuvant chemo-radiation therapy.

Postoperative bleeding was observed in two patients $(2.3 \%)$ in this study. One patient showed bleeding from a branch of the right bronchial artery on postoperative day 2 and required reoperation. Another patient presented with postoperative bleeding from a branch of the right gastroepiploic artery after gastric tube reconstruction on postoperative day 0 and required reoperation. These bleeding events were not related to lymphadenectomy, and the patients were discharged following an extended standard perioperative course. Pneumonia was observed in three patients (3.4\%) in this study, which is a lower incidence than previously reported [2, 3, 6]. Among these three patients, one patient had previous experienced postoperative bleeding, and he developed atelectasis, anastomotic leakage, and pneumonia. Two elderly patients (over 85 years old) developed aspiration pneumonia, which suggests that this technique may limit the development of aspiration pneumonia. We believe that this approach helps limit the incidence of recurrent laryngeal nerve palsy and is a simple technique based on anatomical structures, facilitating its use by most esophageal surgeons.

This study has limitations. It is a single-institution retrospective study, and all operations were performed by a highly experienced team. These results may not be generalizable but are encouraging and support further study of the native tissue preservation technique. These results are expected to contribute to the prevention of recurrent laryngeal nerve palsy during minimally invasive esophagectomy with radical lymph node dissection. 
In conclusion, the native tissue preservation technique maintains the normal anatomical position of the recurrent laryngeal nerve, avoiding stretching, thermal damage, and direct contact to the nerve. This technique was used in 87 consecutive patients who underwent minimally invasive radical esophagectomy with radical lymph node dissection, and there was no incidence of RLNP. This native tissue preservation technique may reduce the incidence of recurrent laryngeal nerve paralysis after minimally invasive esophagectomy with radical lymph node dissection.

Acknowledgements The authors particularly thank Nobuyuki Yajima, M.D., Ph.D., and Kris Siriratsivawong M.D., F.A.C.S., for their help in proofreading the manuscript.

Author contributions Study conception and design: KO, MM, TA. Analysis and interpretation of data: KO, SG, TY, TA, MK, AS, RK, AKL. Drafting of manuscript: KO, MM, TA, AKL. Critical revision: KO, MM, SG, TY, TA, MK, AS, RK, AKL, TA.

Funding None.

\section{Compliance with ethical standards}

Disclosures Drs. Otsuka, Murakami, Goto, Ariyoshi, Yamashita, Saito, Kohmoto, Kato, Lefor, and Aoki have no conflicts of interest or financial ties to disclose.

Open Access This article is licensed under a Creative Commons Attribution 4.0 International License, which permits use, sharing, adaptation, distribution and reproduction in any medium or format, as long as you give appropriate credit to the original author(s) and the source, provide a link to the Creative Commons licence, and indicate if changes were made. The images or other third party material in this article are included in the article's Creative Commons licence, unless indicated otherwise in a credit line to the material. If material is not included in the article's Creative Commons licence and your intended use is not permitted by statutory regulation or exceeds the permitted use, you will need to obtain permission directly from the copyright holder. To view a copy of this licence, visit https://creativecommons.org/licenses/by/4.0/.

\section{References}

1. Bartels H, Stein HJ, Siewert JR (1998) Preoperative risk analysis and postoperative mortality of oesophagectomy for resectable oesophageal cancer. Br J Surg 85:840-844

2. Booka E, Takeuchi H, Nishi T, Matsuda S, Kaburagi T, Fukuda K, Nakamura R, Takahashi T, Wada N, Kawakubo H, Omori T, Kitagawa Y (2015) The impact of postoperative complications on survivals after esophagectomy for esophageal cancer. Medicine 94:e1369

3. Ninomiya I, Osugi H, Fujimura T, Fushida S, Okamoto K, Maruzen S, Oyama K, Kinoshita J, Tsukada T, Kitagawa H, Takamura H, Nakagawara H, Tajima H, Hayashi H, Makino I, Ohta T (2014) Thoracoscopic esophagectomy with extended lymph node dissection in the left lateral position: technical feasibility and oncologic outcomes. Dis Esophagus 27:159-167
4. Takemura M, Hori T, Fujiwara Y (2013) Clinical outcomes and prognosis after thoracoscopic esophagectomy with two-field lymph node dissection for lower thoracic esophageal cancer. Anticancer Res 33:601-608

5. Murakami M, Otsuka K, Goto S, Ariyoshi T, Yamashita T, Aoki T (2017) Thoracoscopic and hand assisted laparoscopic esophagectomy with radical lymph node dissection for esophageal squamous cell carcinoma in the left lateral decubitus position: a single center retrospective analysis of 654 patients. BMC Cancer 17:748

6. Takeuchi H, Miyata H, Ozawa S, Udagawa H, Osugi H, Matsubara H, Konno H, Seto Y, Kitagawa Y (2017) Comparison of shortterm outcomes between open and minimally invasive esophagectomy for esophageal cancer using a nationwide database in Japan. Ann Surg Oncol 24:1821-1827

7. Rusch VW, Rice TW, Crowley J, Blackstone EH, Rami-Porta R, Goldstraw P (2010) The seventh edition of the American Joint Committee on Cancer/International Union against cancer staging manuals: the new era of data-driven revisions. J Thorac Cardiovasc Surg 139:819-821

8. Clavien PA, Strasberg SM (2009) Severity grading of surgical complications. Ann Surg 250:197-198

9. Katayama H, Kurokawa Y, Nakamura K, Ito H, Kanemitsu Y, Masuda N, Tsubosa Y, Satoh T, Yokomizo A, Fukuda H, Sasako M (2016) Extended Clavien-Dindo classification of surgical complications: Japan Clinical Oncology Group postoperative complications criteria. Surg Today 46:668-685

10. Tachimori Y, Ozawa S, Numasaki H, Fujishiro M, Matsubara H, Oyama T, Shinoda M, Toh Y, Udagawa H, Uno T, Registration Committee for Esophageal Cancer of the Japan Esophageal Society (2016) Comprehensive registry of esophageal cancer in Japan, 2009. Esophagus 13:110-137

11. Akiyama H, Tsurumaru M, Udagawa H, Kajiyama Y (1994) Radical lymph node dissection for cancer of the thoracic esophagus. Ann Surg 220:364-372

12. Tsurumaru M, Kajiyama Y, Udagawa H, Akiyama H (2001) Outcomes of extended lymph node dissection for squamous cell carcinoma of the thoracic esophagus. Ann Thorac Cardiovasc Surg 7:325-329 (Discussion 372-373)

13. Udagawa $H$, Ueno M, Shinohara $H$, Haruta $S$, Kaida S, Nakagawa M, Tsurumaru M (2012) The importance of grouping of lymph node stations and rationale of three-field lymphoadenectomy for thoracic esophageal cancer. J Surg Oncol 106:742-747

14. Koch C, Friedrich T, Metternich F, Tannapfel A, Reimann HP, Eichfeld U (2003) Determination of temperature elevation in tissue during the application of the harmonic scalpel. Ultrasound Med Biol 29:301-309

15. Humes DJ, Ahmed I, Lobo DN (2010) The pedicle effect and direct coupling: delayed thermal injuries to the bile duct after laparoscopic cholecystectomy. Arch Surg 145:96-98

16. Perko Z, Pogorelic Z, Bilan K, Tomić S, Vilović K, Krnić D, Druzijanić N, Kraljević D, Juricić J (2006) Lateral thermal damage to rat abdominal wall after harmonic scalpel application. Surg Endosc 20:322-324

17. Emam TA, Cuschieri A (2003) How safe is high-power ultrasonic dissection? Ann Surg 237:186-191

18. Diamantis T, Kontos M, Arvelakis A, Syroukis S, Koronarchis D, Papalois A, Agapitos E, Bastounis E, Lazaris AC (2006) Comparison of monopolar electrocoagulation, bipolar electrocoagulation, ultracision, and ligasure. Surg Today 36:908-913

19. Campbell PA, Cresswell AB, Frank TG, Cuschieri A (2003) Real-time thermography during energized vessel sealing and dissection. Surg Endosc 17:1640-1645

20. Karle H, Hansen NE (1970) Changes in the red cell membrane induced by a small rise in temperature. Scand J Clin Lab Invest 26:169-174 
21. Sutton PA, Awad S, Perkins AC, Lobo DN (2010) Comparison of lateral thermal spread using monopolar and bipolar diathermy, the Harmonic Scalpel and the Ligasure. Br J Surg 97:428-433

22. Applewhite MK, White MG, James BC, Abdulrasool L, Kaplan EL, Angelos P, Grogan RH (2017) Ultrasonic, bipolar, and integrated energy devices: comparing heat spread in collateral tissues. J Surg Res 207:249-254

23. Gardner IH, Doherty GM, McAneny D (2016) Intraoperative nerve monitoring during thyroid surgery. Curr Opin Endocrinol Diabetes Obes 23:394-399

24. Schneider R, Randolph GW, Barczynski M, Dionigi G, Wu CW, Chiang FY, Machens A, Kamani D, Dralle H (2016) Continuous intraoperative neural monitoring of the recurrent nerves in thyroid surgery: a quantum leap in technology. Gland Surg 5:607-616

25. Brauckhoff K, Aas T, Biermann M, Husby P (2017) EMG changes during continuous intraoperative neuromonitoring with sustained recurrent laryngeal nerve traction in a porcine model. Langenbecks Arch Surg 402:675-681

26. Deguchi T, Ikeda Y, Niimi M, Fukushima R, Kitajima M (2017) Continuous intraoperative neuromonitoring study using pigs for the prevention of mechanical recurrent laryngeal nerve injury in esophageal surgery. Surg Innov 24:115-121

27. Ismail HEA, Kasem MA, Mostafa FE (2017) Influence of blood flow on the neuroma formation after transposition of the nerve stump into vein: experimental and clinical study. J Hand Microsurg 9:17-27

28. Takamori Y, Arimizu J, Izaki T, Naito M, Kobayashi T (2011) Combined measurement of nerve root blood flow and electrophysiological values: intraoperative straight-leg-raising test for lumbar disc herniation. Spine 36:57-62

29. Bedenne L, Michel P, Bouché O, Milan C, Mariette C, Conroy T, Pezet D, Roullet B, Seitz JF, Herr JP, Paillot B, Arveux P, Bonnetain F, Binquet $C$ (2007) Chemoradiation followed by surgery compared with chemoradiation alone in squamous cell cancer of the esophagus: FFCD 9102. J Clin Oncol 25:1160-1168

Publisher's Note Springer Nature remains neutral with regard to jurisdictional claims in published maps and institutional affiliations. 